Tropical Journal of Pharmaceutical Research September 2021; 20 (9): 1833-1838

ISSN: $1596-5996$ (print); 1596-9827 (electronic)

(C) Pharmacotherapy Group, Faculty of Pharmacy, University of Benin, Benin City, 300001 Nigeria.

\title{
Upregulation of tetraspanin 8 may contribute to LPS- induced acute lung injury by activation of the MAPK and NF-KB pathways
}

\author{
Youcai Zhu ${ }^{1}$, Feng Tao ${ }^{2}$, Xiaolong $\mathrm{Ma}^{2}$, Junhua Guo ${ }^{3}$, Tian Tian ${ }^{4}$, Shiping Zhu ${ }^{5}$, \\ Yongzhi Feng ${ }^{2 *}$ \\ ${ }^{1}$ Department of Thoracic Disease Center, Zhejiang Rongjun Hospital, Jiaxing, Zhejiang Province 314000, ${ }^{2}$ Department of \\ Respiratory Medicine, The First Hospital of Jiaxing (The Affiliated Hospital of Jiaxing University), Jiaxing, Zhejiang Province \\ 314001, ${ }^{3}$ Department of Oncology, Hangzhou Hospital of Traditional Chinese Medicine, Hangzhou, Zhejiang Province, 310007, \\ ${ }^{4}$ Department of Oncology, The First Affiliated Hospital of Zhejiang University Medical College, Hangzhou, Zhejiang Province \\ 310000, ${ }^{5}$ Department of Respiratory and Critical Care Medicine. Hangzhou Hospital of Traditional Chinese Medicine, \\ Hangzhou, Zhejiang Province 310007, China
}

*For correspondence: Email: fengzhiyong_666@163.com; Tel: +86- 0573-82082937

Sent for review: 4 June 2021

Revised accepted: 26 August 2021

\begin{abstract}
Purpose: To investigate the effect of tetraspanin8 (Tspan8, also known as TM4SF3 or CO-029) on lipopolysaccharide (LPS)-induced acute lung injury (ALI) and the related signaling pathways.

Methods: Treatment with LPS was used to induce lung damage in mice and a lung epithelial cell line. The wet-to-dry weight ratio of lung tissue, hematoxylin and eosin (H\&E) staining, and quantification of cytokine concentrations were conducted to validate the model. Enzyme-linked immunosorbent assays (ELISA) and quantitative polymerase chain reaction (qPCR) were used to measure levels of tumor necrosis factor alpha, interleukin (IL)-1 $\beta$, and IL-6. Tspan8 levels were knocked down using shRNAs. Mitogen-activated protein kinase (MAPK) and nuclear factor kappa B (NF-KB) pathway levels were assessed after LPS-induced injury in this cellular model.

Results: Levels of Tspan8 were upregulated in the LPS-induced ALI model. Additionally, LPS treatment of mouse lung epithelial cells resulted in Tspan8 upregulation. Tspan8 knockdown alleviated the effects of LPS on lung epithelial injury by inhibiting the upregulation of MAPK and NF-KB signaling pathways. Conclusion: The upregulation of Tspan 8 may promote the progression of ALI.
\end{abstract}

Keywords: Acute lung injury (ALI), Tetraspanin 8 (Tspan8), LPS, MAPK, NF-KB

This is an Open Access article that uses a funding model which does not charge readers or their institutions for access and distributed under the terms of the Creative Commons Attribution License (http://creativecommons.org/licenses/by/4.0) and the Budapest Open Access Initiative (http://www.budapestopenaccessinitiative.org/read), which permit unrestricted use, distribution, and reproduction in any medium, provided the original work is properly credited.

Tropical Journal of Pharmaceutical Research is indexed by Science Citation Index (SciSearch), Scopus, International Pharmaceutical Abstract, Chemical Abstracts, Embase, Index Copernicus, EBSCO, African Index Medicus, JournalSeek, Journal Citation Reports/Science Edition, Directory of Open Access Journals (DOAJ), African Journal Online, Bioline International, Open-J-Gate and Pharmacy Abstracts

\section{INTRODUCTION}

Acute lung injury (ALI) involves injury to both alveolar epithelial cells and pulmonary capillary endothelial cells caused by non-cardiac factors [1]. Pathological features of ALI include impaired alveolar capillary barriers, diffuse alveolar, and pulmonary interstitial edema [2]. Additionally, the main pathophysiological features of $\mathrm{ALI}$ are decreased lung compliance and decreased lung volume [3]. The causes of ALI include shock, trauma, pneumonia, and other internal and 
external pulmonary factors [4]. Respiratory distress and refractory hypoxemia are clinical characteristics of ALI that can worsen without intervention [2]. Despite progress in the diagnosis and treatment of ALI in recent years, the disease still has a high mortality rate of 38.5 $\%$ [3]. To improve treatment and outcomes for patients with ALI, detailed studies of its pathogenesis are needed, including the related regulatory proteins.

Although multiple pathologies can induce the onset of ALI, such as sepsis, pneumonia, gastric content inhalation, severe trauma, and acute pancreatitis, gram-negative bacteria-induced sepsis is the most common [4] [5]. While the advantages of lipopolysaccharide (LPS)-induced ALI for research include high repeatability and sensitivity, the main disadvantage is that LPS may contain impurities [5]. Therefore, the concentration of LPS and the route of administration should be carefully researched when building relevant animal models.

Tetraspanin 8 (Tspan8, also known as TM4SF3 or CO-029), a member of transmembrane 4 superfamily proteins, has been studied for its role in the progression and metastasis of a wide variety of cancers [6][7]. High expression of Tspan8 in several types of cancers, such as esophageal cancer and liver cancer, correlated with the prognosis [8]. Tspan8 is also a novel therapeutic target in cancer for monoclonal antibody therapy [8]. However, the role of Tspan8 in the progression and pathogenesis of $\mathrm{ALI}$ is still unclear.

In this study, LPS was used to generate an ALI model in mice and cell culture, and a correlation between the upregulation of Tspan8 and LPSinduced ALI was observed. These data further confirm a critical role of Tspan8 in the progression of ALI.

\section{EXPERIMENTAL}

\section{Animals}

The animal experiments were conducted in accordance with the National Institutes of Health Laboratory Animal Care and Use Guidelines and World Medical Association Declaration of Helsinki. Animal studies in this paper were performed according to the guidance of the Ethics Committee of The First Hospital of Jiaxing (approval no. LS2020-008). Mice were purchased from Slac (Shanghai, China) and housed in Plexiglass cages at a temperature of $22 \pm 1^{\circ} \mathrm{C}$ with $\mathrm{a} 12 \mathrm{~h}$ dark-light cycle. The mice had free access to feed and water.

\section{LPS-induced acute lung injury}

Mice were administrated with $100 \mathrm{mg} / \mathrm{kg}$ ketamine and $5 \mathrm{mg} / \mathrm{kg}$ xylazine by intraperitoneal injection. Then, the mice were intratracheally instilled with $1 \mathrm{mg} / \mathrm{kg}$ LPS (E. coli 055:B5, sigma, St. Louis, MO, USA) for $24 \mathrm{~h}$. The lungs were excised, weighed immediately, and then heated in an oven at $60^{\circ} \mathrm{C}$ for 3 days before measuring complete dry weight.

\section{H\&E staining}

Freshly excised lung tissues were fixed with 10 $\%$ formalin, embedded with paraffin, and sliced into $5 \mu \mathrm{m}$ sections. The sliced sections were deparaffinized, and treated with hematoxylin and eosin (Sigma-Aldrich). To evaluate the extent of lung injury, grades were given for the following pathological features: 0 for normal lung, 1 for congestion, 2 for edema, 3 for interstitial inflammation, and 4 for inflammatory cell infiltration. Individual scores for each group were added together to calculate total lung injury score.

\section{Enzyme-linked immunosorbent assays (ELISA)}

The lungs were lavaged with $1 \mathrm{ml} \mathrm{Ca}{ }^{2+} / \mathrm{Mg}^{2+}$ free HBSS after sacrificing the mice. The collected solution was centrifuged at 3,000 rpm for $5 \mathrm{~min}$ and the pellet was discarded. Concentrations of tumor necrosis factor alpha (TNF- $\alpha$ ), interleukin (IL) $-1 \beta$, and IL-6 in bronchoalveolar lavage fluid (BALF) were measured using ELISA Quantikine kit (R \& D Systems).

\section{Immunoblot analysis}

Protein samples from tissues and cells were extracted using lysis buffer (Beyotime, China) and separated through electrophoresis on sodium dodecylsulfate-polyacrylamide gels and transferred to polyvinylidene difluoride membranes. After blocking with $5 \%$ milk in TBST (20 mM Tris, 500mM NaCl, pH 7.5, 0.1\% Tween 20) for $1 \mathrm{~h}$ at room temperature, the membranes were incubated with either antiTspan8 antibody (1:1000), anti-GAPDH (1:10000), anti-IKBa(1:1000), anti-p-IкBa (1:1000), anti-p65 (1:2000), anti-p-p65 (1:1000), anti-ERK (1:1000), or anti-p-ERK $(1: 1000)$ at $4^{\circ} \mathrm{C}$ overnight. The membranes subsequently were washed in TBST 3 times and incubated with secondary antibody with horseradish peroxidase (Sigma) at room temperature for $1 \mathrm{~h}$. The membranes were analyzed with $E C L$ reagent (Sigma).

Trop J Pharm Res, September 2021; 20(9): 1834 
Cell culture and Cell Counting Kit-8 (CCK-8) assay

Mouse lung epithelia cells, MLE-12, were cultured in DMEM/Ham's F-12 (HyClone, USA) supplemented with supplied with $10 \%$ FBS (Biological Industries, Israel) and $1 \%$ penicillin/streptomycin (Biological Industries, Israel) at $37{ }^{\circ} \mathrm{C}$. The MLE-12 cells were seeded into 96-well plates, incubated for $24 \mathrm{~h}$, then treated with LPS at concentrations of either 0.1 , 0.2 , or $0.5 \mu \mathrm{g} / \mathrm{mL}$ for $24 \mathrm{~h}$. For the CCK-8 assay, cells treated with LPS were supplemented with CCK-8. Cell concentrations were assayed with a microplate reader with an absorbance of $490 \mathrm{~nm}$.

\section{Tspan8 knockdown}

Plasmids containing either Tspan8 shRNA or a scrambled shRNA were obtained from Santa Cruz Biotechnology, Inc. and introduced into MLE-12 cells with Lipofectamine 3000 (Invitrogen, Carlsbad, CA, USA) according to the manufacturer's protocol. A cell line with a stable knockdown of Tspan8 was generated by selection with $10 \mu \mathrm{g} / \mathrm{mL}$ of puromycin for 2 weeks. Single clones were isolated and cultured.

\section{Flow cytometry}

Cells were placed into 96-well plate and treated as indicated in the figure legends. Then the cells were fixed and labeled with $50 \mu \mathrm{g} / \mathrm{mL}$ propidium iodide at room temperature for $30 \mathrm{~min}$ in the dark. The treated cells were washed using centrifugation, resuspended and analyzed on a BD FACSCanto II (BD Biosciences).

\section{Quantitative PCR}

Total RNA was extracted using TRIzol (Invitrogen, Waltham, MA, USA). RNA was reverse-transcribed into CDNA using Moloney Murine Leukemia Virus Reverse Transcriptase (Promega, Madison, WI, USA). Fast Start Universal SYBR Green Master kit (Roche, Basel, Switzerland) was used for quantitative mRNA detection on ABI StepOne system (Applied BioSystems, Foster City, CA, USA). The levels of targeted genes were determined using the $2^{-\triangle \triangle C T}$ method [9]. The primers used are listed in Table 1.

\section{Statistical analyses}

Data are presented as mean \pm standard deviation. The data was analyzed with GraphPad Prism. $p$ values were derived by Student's t test and a value $<0.05$ was considered statistically significant.

\section{RESULTS}

\section{Tspan8 was upregulated in LPS-induced ALI in mice}

Intratracheal injections of LPS were used to model ALI in mice. The ratio of wet weight to dry weight of the lung tissue weight and $\mathrm{H} \& \mathrm{E}$ staining were used to assess ALI. After LPS induction, the wet-to-dry ratio of lung tissue weight increased (Figure 1A). In the LPS-induced lung injury group, histological analysis revealed LPS administration induced ALI (Figure 1B). After LPS treatment, concentrations of TNF- $\alpha$, IL$1 \beta$, and IL- 6 were significantly increased (Figure 1C). Furthermore, Tspan8 protein levels significantly increased in the LPS injected group compared to controls (Figure 1D). These findings indicate that Tspan8 levels are elevated in LPSinduced ALI.
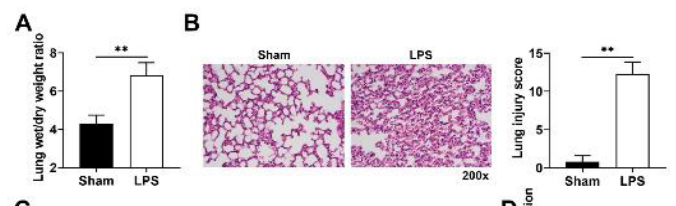

\section{C}
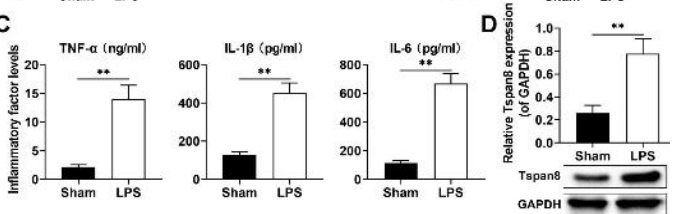

Figure 1: Tspan8 is upregulated in LPS-induced ALI in mice. (A) The lung wet-to-dry weight ratio in Sham and LPS treated mice. (B) H\&E staining of lung tissue in Sham and LPS-treated mice. (C) IL-6, IL-1 $\beta$, and TNF- $\alpha$ concentrations in BALF of mice treated with Sham or LPS. (D) Immunoblot assay and quantification of the levels of Tspan8 in Sham and LPS-treated mice. ${ }^{* *} p<0.01$

Table 1: Primers for quantitative PCR

\begin{tabular}{lll}
\hline Genes & Forward primers & Reverse primers \\
\hline TNF- $\alpha$ & GAACTGGCAGAAGAGGCACT & GGTCTGGGCCATAGAACTGA \\
IL-6 & CTGATGCTGGTGACAACCAC & CAGAATTGCCATTGCACAAC \\
IL-1 $\beta$ & TGGACCTTCCAGGATGAGGAC & GTTCATCTCGGAGCCTGTAGTG \\
GAPDH & AGTATGACTCCACTCACGGC & CACCAGTAGACTCCACGACA \\
\hline
\end{tabular}


Elevated Tspan8 levels in LPS-induced MLE12 cells

MLE-12 cells were used to further confirm the changes in protein levels of Tspan8 after LPS induction. Treatment with increasing concentrations of LPS reduced MLE-12 cell viability in a dose dependent manner (Figure 2A). Additionally, LPS treatment increased cell apoptosis compared to controls (Figure 2B). After LPS treatment, TNF- $\alpha$, IL- $1 \beta$, and IL- 6 concentrations were significantly increased in MLE-12 cells (Figure 2C). Furthermore, Tspan8 protein levels increased after LPS induction in MLE-12 cells (Figure 2D). These results suggest that LPS can induce Tspan8 expression in MLE12 cells.

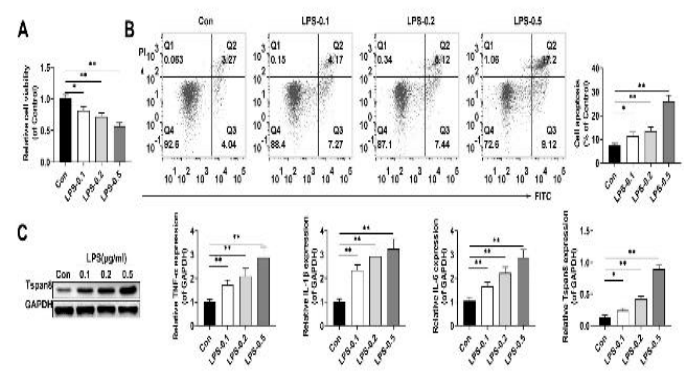

Figure 2: Tspan8 is elevated in LPS treated MLE-12 cells. (A) Relative cell viability of MLE-12 cells with or without LPS addition. (B) Detection of cell death in MLE-12 cell populations treated with varied concentrations of LPS. (C) IL-6, IL-1 $1 \beta$, and TNF- $\alpha$ mRNA levels in MLE-12 cells treated with varied concentrations of LPS. (D) Immunoblot assay detected the level of Tspan8 in MLE-12 cells treated with varied concentrations of LPS. ${ }^{* *} p<0.01$

\section{Tspan8 knockdown alleviates LPS-induced lung epithelial cell injury}

To explore the potential role of Tspan8 expression in lung epithelial cells, Tspan8 levels were depleted in MLE-12 cells. Immunoblot assays confirmed the efficiency of shRNA knockdown of Tspan8 in MLE-12 cells (Figure 3A). Tspan8 knockdown ameliorated both the decreased cell viability and increased cell death observed after LPS treatment (Figure 3B, C). Knockdown of Tspan8 also blocked the secretion of IL-6, IL-1 $\beta$, and TNF- $\alpha$ cytokines induced by LPS in lung epithelial cells (Figure 3D). These data suggest Tspan8 depletion can prevent LPSinduced damage to lung epithelial cells.

Tspan8 knockdown inhibits MAPK and NF-kB signaling pathway

Mitogen-activated protein kinase (MAPK) and nuclear factor kappa B (NF-KB) signaling pathways are vital in LPS-induced ALI [10]. Moreover, LPS stimulation induces phosphorylation of MAPKs, including ERK, p38 MAPK, and JNK pathways. The addition of LPS to MLE-12 cells activated MAPK and NF-kB. LPS treatment effectively enhanced $p-1 k B-\alpha, p-p 65, p-$ ERK and p-p38 expression (Figure 4). However, Tspan8 knockdown significantly inhibited p-lkB$\alpha, p-p 65, p-E R K$, and p-p38 expression (Figure 4). Interestingly, after LPS treatment IkB- $\alpha$ levels were reduced, but Tspan8 knockdown elevated IkB- $\alpha$ levels (Figure 4). These findings suggest that Tspan8 knockdown inhibits MAPK and NF$\mathrm{kB}$ signaling pathways.

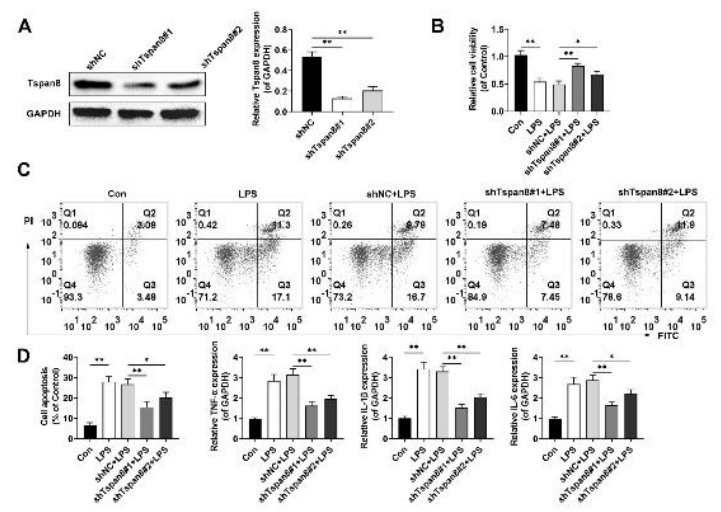

Figure 3: Tspan8 knockdown alleviates LPS-induced lung epithelial cell injury. (A) Immunoblot assay of Tspan8 knockdown in MLE-12 cells. (B) Relative cell viability in control, LPS, and Tspan8 knockdown in LPS-treated MLE-12 cells. (C) Cell apoptosis in control, LPS, and Tspan8 knockdown with LPS-treated MLE-12 cells. (D) IL-6, IL-1 $\beta$, and TNF- $\alpha$ mRNA levels in control, LPS, and Tspan8 knockdown in LPStreated MLE-12 cells. ${ }^{*} p<0.05,{ }^{* *} p<0.01$

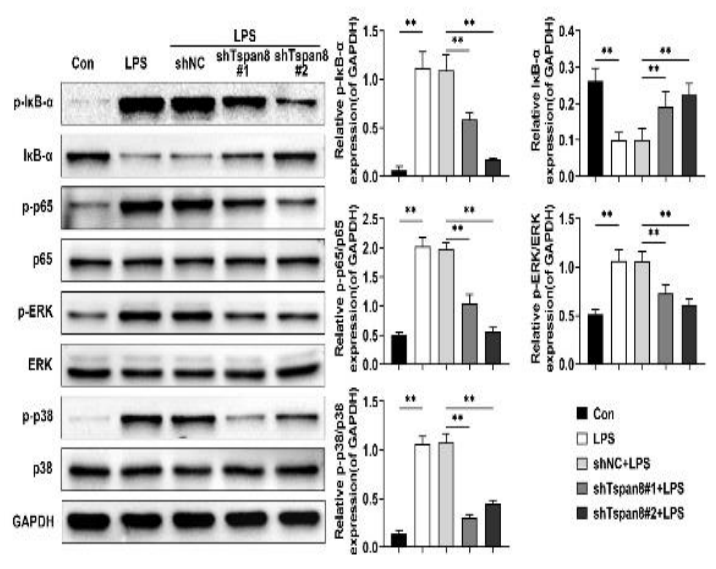

Figure 4: Tspan8 knockdown inhibits MAPK and NF-kB signaling pathways. Immunoblot assay of protein levels of $p$-lkB- $\alpha$, IkB- $\alpha, p-p 65, p 65, p-$ ERK, ERK, and p-p38 in control, LPS, and Tspan8 knockdown in LPS-treated MLE-12 cells. ${ }^{* *} p<0.01$ 


\section{DISCUSSION}

ALI can lead to acute respiratory distress syndrome, multiple organ dysfunction syndrome, or even death in severe cases [11]. ALI pathogenesis is usually due to injury of alveolar epithelial cells and capillary endothelial cells [12]. ALI can be initiated by multiple factors, such as pneumonia, pulmonary aspiration, inhalation of toxic gases, and ischemia-reperfusion injury [13]. Elucidation of ALI pathogenesis and identification of the key proteins that regulate its progression are needed to improve the survival rate of patients. In this study, a transmembrane 4 superfamily protein, Tspan8, was found to be upregulated in both an LPS-induced ALI mice model and LPS-treated mouse lung epithelial cells. Interestingly, the depletion of Tspan8 resulted in the alleviation of the observed injury to lung epithelial cells after LPS treatment. Therefore, Tspan8 is likely involved in the progression of ALI.

Confirmed by immunohistochemistry, this study successfully established a mouse model of ALI using LPS treatment. Advantages of this model include increased reproducibility and decreased cost compared to other models. Furthermore, LPS has less direct toxicity to cells in vitro. Disadvantages of this model include the fact that LPS usually contains pollutants, which can affect its biological activity. This model can be used for subsequent gene function analysis and phenotype detection. The sensitivity and the route of administration of LPS in laboratory animals was very important for successful construction of this model.

Previous work has found that blocking toll-like receptor 4 (TLR4) or using TLR4-knockout mice in the LPS-induced ALI model can significantly suppress the NF-kB signaling pathway, reduce the release of inflammatory mediators, and reduce the degree of lung injury [14]. Together, this suggests that inhibition of inflammatory signaling pathways can alleviate lung injury. In further support of this conclusion, this study found that knockdown of Tspan8 can slow down lung injury through the inhibition of inflammation related signal pathways, including MAPK and NF-KB.

As a member of the transmembrane 4 superfamily proteins, Tspan8 has been reported not only to play an important role in both the occurrence and development of a variety of tumors but also to affect tumor progression through different regulatory mechanisms and signaling pathways [15]. For example, Tspan8 drives dermal invasion by melanoma cells via contributing to the activation of promatrix metalloproteinase- 9 and the proteolysis of the basement membrane in a keratinocytedependent manner [16].

Tspan8 could promote colorectal cancer cell growth and migration in lysine-specific demethylase 1-dependent manner or via $\beta$ catenin pathways. However, there are few reports on its non-tumor related functions. A previous study showed that Tspan8 could mediate endothelial cell and fibroblast remodeling, which mainly relied on the target cell-selective response [17]. Joint features and complementarities of Tspan8 and CD151 were also revealed [18]. In this study, Tspan8 depletion alleviated LPS-induced lung epithelial injury in mice via suppression of MAPK and NF$\mathrm{KB}$ signaling pathways. However, the precise molecular mechanisms need further study.

\section{CONCLUSION}

Levels of Tspan8 are elevated in a LPS-induced ALI mouse model, as well as in LPS-treated mouse lung epithelial cells. Furthermore, Tspan8 knockdown alleviates LPS-induced lung epithelial injury and suppresses MAPK and NF-kB signaling pathways. Thus, these data confirm the critical role of Tspan8 in the progression of ALI.

\section{DECLARATIONS}

\section{Conflict of interest}

No conflict of interest is associated with this work.

\section{Contribution of authors}

We declare that this work was done by the authors named in this article and all liabilities pertaining to claims relating to the content of this article will be borne by the authors. Youcai Zhu and Feng Tao designed the study and supervised the data collection; Xiaolong Ma and Junhua Guo analyzed and interpreted the data; and Tian Tian, Shiping Zhu, and Yongzhi Feng prepared the manuscript for publication and reviewed the draft of the manuscript. All authors have read and approved the manuscript.

\section{Open Access}

This is an Open Access article that uses a funding model which does not charge readers or their institutions for access and distributed under the terms of the Creative Commons Attribution License (http://creativecommons.org/licenses/by/ 
4.0) and the Budapest Open Access Initiative (http://www.budapestopenaccessinitiative.org/rea d), which permit unrestricted use, distribution, and reproduction in any medium, provided the original work is properly credited.

\section{REFERENCES}

1. Lu Z, Yang H, Cao H, Huo C, Chen Y, Liu D, Xie P, Zhou $H$, Liu J, Yu L. Forsythoside A protects against lipopolysaccharide-induced acute lung injury through upregulating microRNA-124. Clin Sci 2020; 134(19): 25492563.

2. Pourfathi M, Kadlecek SJ, Chatterjee S, Rizi RR. Metabolic Imaging and Biological Assessment: Platforms to Evaluate Acute Lung Injury and Inflammation. Front Physiol 2020; 11.

3. van der Laan EAZ, van der Velden S, Porcelijn L, Semple $J W$, van der Schoot CE, Kapur R. Update on the pathophysiology of transfusion-related acute lung injury. Curr Opin Hematol 2020; 27(6): 386-391.

4. Zhao $X, G u$ C, Wang Y. PAD4 selective inhibitor TDFA protects lipopolysaccharide-induced acute lung injury by modulating nuclear p65 localization in epithelial cells. Int Immunopharmacol 2020; 88: 106923.

5. Lu Z-b, Liu S-h, Ou J-y, Cao H-h, Shi L-z, Liu D-y, Tian C-y, Zheng Y-r, Zhou H-I, Liu J-s. Forsythoside A inhibits adhesion and migration of monocytes to type II alveolar epithelial cells in lipopolysaccharide-induced acute lung injury through upregulating miR-124. Toxicol Appl Pharm 2020; 407: 115252.

6. Iwai K, Ishii M, Ohshima S, Miyatake K, Saeki Y. Expression and function of transmembrane-4 superfamily (tetraspanin) proteins in osteoclasts: reciprocal roles of Tspan-5 and NET-6 during osteoclastogenesis. Allergol Int 2007; 56(4): 457-463.

7. Cai $Y$, Zheng $M$, Zhao Z, Huang $H$, Fu $W, X u X$. Expression of Tspan-1 gene in patients with advanced gastric cancer. Oncol Lett 2017; 14(3): 2996-3000.

8. Maisonial-Besset A, Witkowski $T$, Navarro-Teulon I, Berthier-Vergnes O, Fois G, Zhu Y, Besse S, Bawa O, Briat A, Quintana M. Tetraspanin 8 (TSPAN 8) as a potential target for radio-immunotherapy of colorectal cancer. Oncotarget 2017; 8(13): 22034.

9. Jin Y, Tachibana I, Takeda Y, He P, Kang S, Suzuki M, Kuhara $H$, Tetsumoto S, Tsujino K, Minami $T$, et al.
Statins decrease lung inflammation in mice by upregulating tetraspanin CD9 in macrophages. PLoS One 2013; 8(9): e73706.

10. Chen $X$, Yang $X$, Liu T, Guan M, Feng $X$, Dong $W$, Chu $X$, Liu J, Tian $X, C i X$, et al. Kaempferol regulates MAPKs and NF-kappaB signaling pathways to attenuate LPS-induced acute lung injury in mice. Int Immunopharmacol 2012; 14(2): 209-216.

11. Wen $H$, Zhang H, Wang W, Li Y. Tetrahydropalmatine protects against acute lung injury induced by limb ischemia/reperfusion through restoring PI3KJAKT/mTOR-mediated autophagy in rats. Pulm Pharmacol Ther 2020; 64: 101947.

12. Badamjav R, Zhang L, Sonom D, Wu YH, Kou JP, Yu $B Y$, Li F. Thalictrum minus $L$. ameliorates particulate matter-induced acute lung injury in mice. J Ethnopharmacol 2020; 264: 113379.

13. Huang Z, Zheng J, Huang W, Yan M, Hong L, Hong Y, Jin $R$, Huang $X$, Fan $H$, Chen $H$, et al. The effects and safety of omega-3 fatty for acute lung injury: a systematic review and meta-analysis. World J Surg Oncol 2020; 18(1): 235.

14. Yang $R$, Hu $X$, Xie $X$, Chen $H$, Fang $H$, Zhu L, Li Z. Propionic Acid Targets the TLR4/NF-kappaB Signaling Pathway and Inhibits LPS-Induced Intestinal Barrier Dysfunction: In Vitro and In Vivo Studies. Front Pharmacol 2020; 11: 573475.

15. Lin X, Bi Z, Hu Q, Li Q, Liu J, Luo ML, Xiang Y, Yao H. TSPAN8 serves as a prognostic marker involving Akt/MAPK pathway in nasopharyngeal carcinoma. Ann Transl Med 2019; 7(18): 470.

16. El Kharbili M, Cario M, Bechetoille N, Pain C, Boucheix C, Degoul F, Masse I, Berthier-Vergnes O. Tspan8 Drives Melanoma Dermal Invasion by Promoting ProMMP-9 Activation and Basement Membrane Proteolysis in a Keratinocyte-Dependent Manner. Cancers (Basel) 2020; 12(5).

17. Mu W, Provaznik J, Hackert T, Zoller M. Tspan8-Tumor Extracellular Vesicle-Induced Endothelial Cell and Fibroblast Remodeling Relies on the Target CellSelective Response. Cells 2020; 9(2).

18. Yue S, Mu W, Erb U, Zoller M. The tetraspanins CD151 and Tspan8 are essential exosome components for the crosstalk between cancer initiating cells and their surroundings. Oncotarget 2015; 6(4): 2366-2384. 